\title{
Coordinated Design of PSS withMultiple FACTS Controllersusing Advanced Adaptive PSO
}

\author{
Rajendraprasad Narne and Prafulla Chandra Panda \\ Department of Electrical Engineering, National Institute of Technology, Rourkela, Orissa, India \\ nrajnitrkl@gmail.com
}

\begin{abstract}
This study presents coordinated control tuning of power system stabilizer (PSS) with series and shunt FACTS controllers. Here thyristor controlled series compensator (TCSC) and static synchronous compensator (STATCOM) based controllers are coordinated to enhance the damping of power oscillations and to improve voltage stability. The design of proposed damping controller is formulated as an optimization problem and the controller gains of a non-linear power system are optimized instantaneously using advanced adaptive particle swarmoptimization (AAPSO). Here the power system employed with PSS,TCSC and STATCOM. To compare the performance of proposed controller four different control schemes employed on test power system. Finally, the proposed coordinated controller performance is discussed with time domain simulations. Wide ranges of loading conditions are employed on the test system to test the robustness of proposed coordinate controller and the simulation results are compared with different control schemes.
\end{abstract}

Keywords: Coordinated design, Particle swarmoptimization, STATCOM, PSS, TCSC.

\section{Introduction}

Power oscillations damping in power system is one of the major challenges to the electrical utilities. Stability (damping) of these oscillations depends on the strength of the transmission system as seen by the power plant, generator excitation control systems and plant output [1]. In order to damp these power system oscillations and increase system stability, one of the conventional, economical and effective solutions is to install the power system stabilizer (PSS) [2-3]. However, the use of PSSs only may not be, in some cases, effective in providing sufficient damping for power oscillations, specifically for long distance power transmission.

The concept of flexible AC transmission systems (FACTS) has been feasible due to the application of high-power electronic devices for power flow, voltage control, and additionally enhancing the damping of power oscillations [4]. Among the FACTS controllers, series compensation devices like thyristor controlled series compensator (TCSC) and Shunt compensation devices like static synchronous compensator (STATCOM) are robust devices in view of power system dynamic stability point. TCSC is thyristor based controller and STATCOM is voltage source converter based controller. By controlling the effective reactance, the TCSC will control the power transfer. On the other hand, STATCOM controls the magnitude of voltage so that power exchanges between the STATCOM and the transmission line [5].

Particle Swarm Optimization (PSO) technique, developed by Kennedy and Eberhart [6], is found applicability and has been used extensively in solving various problems in power systems. Introduction of PSO to search for optimal settings of rule based PSS have been discussed in [7]. Multi-objective design of multi-machine power system stabilizers using particle swarm optimization (PSO) is proposed in [8]. Power system stability enhancement via excitation and FACTS-based stabilizers is thoroughly investigated in [9]. Here, eigenvaluebased objective function to increase the system damping and improve the system response is developed and it is optimized using real-coded genetic algorithm (GA). However, from an evolutionary point of view, the performance of the PSO is better than that of GA [10] and the

Received: April $8^{\text {th }}, 2013$. Accepted: September $2^{\text {nd }}, 2013$ 
authors claimed that PSO arrives at its final parameter values in fewer generations than the GA. Moreover the authors tested several stabilizers like PSS, SVC, TCSC and TCPS individually to enhance system stability.

In literature several researchers proposes the coordination of PSS with FATCS controllers to enhance dynamic performance of the power system. In [11], the authors discussed global tuning procedure for PSS and FACTS devices using a parameter-constrained nonlinear optimization algorithm. A robust coordinated design of a PSS and TCSCbased stabilizer is thoroughly investigated in [12], here an eigenvalue-based objective function is optimized using GA. In [13] the authors develop a novel algorithm for simultaneous coordinated designing of PSS and TCSC based controllers using bacterial swarm optimization. In [14], a multivariable design method has been performed on coordination between internal $\mathrm{AC}$ and $\mathrm{DC}$ voltage controllers. The coordination between the AC and DC voltage PI controllers was taken into consideration. A combination of a relay-switched shunt capacitor and a voltage-sourced inverter based static synchronous compensator to enhance dynamic compensation capability is discussed in [15]. In spite of this, eigenvalue-based control coordination design for achieving optimal damping of the electromechanical oscillations is discussed [16-17].

The present work deals with simultaneous coordinate tuning of PSS, TCSC and STATCOM based controllers. The TCSC damping controller improves the transient stability and STATCOM damping controller improves the voltage stability of the system. Hence, the combination of these controllers can enhance the overall stability of power system. Here, the control parameters of coordinate controller are optimized by minimizing objective function using AAPSO. The proposed controllers are tested individually as well as simultaneously with time-domain simulations. The robustness of the proposed controllers to enhance the power system dynamic performance is tested under different loading conditions.

\section{Modelling of Power System with PSS}

A. Synchronous Machine Modelling

The SMIB system equipped with PSS for generator excitation and FACTS based damping controllers are installed at generator terminals shown in Figure1. The coordinated controller shown in Figure 1 gives the control signals to all three stabilizers. The generator is modeled in third order model [2], which consists of electromechanical swing equation, and q-axis generator internal voltage equation. The dynamic equations are shown equations (1)-(3).

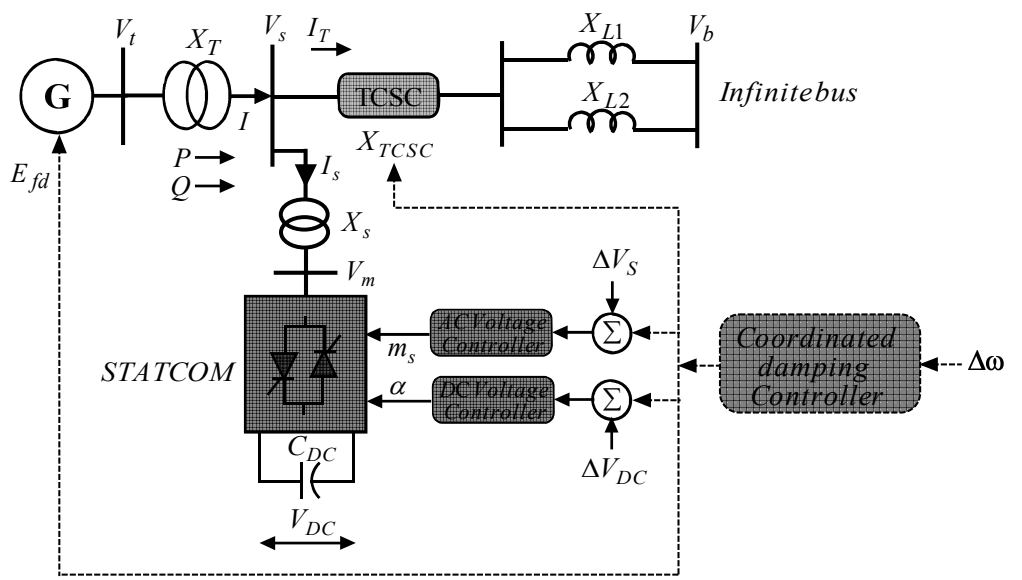

Figure 1. PSS and FACTS stabilizers connected to SMIB system

$$
\dot{\delta}=\omega(t)-\omega_{0}
$$




$$
\begin{aligned}
& \dot{\omega}=-\frac{D}{2 H}\left[\omega(t)-\omega_{0}\right]+\frac{\omega_{0}}{2 H}\left[P_{m}-P_{e}(t)\right] \\
& \dot{E_{q}^{\prime}}=\frac{1}{T_{d 0}^{1}}\left[E_{f d}(t)-\left(X_{d}-X_{d}^{\prime}\right) I_{d}(t)-E_{q}^{\prime}(t)\right]
\end{aligned}
$$

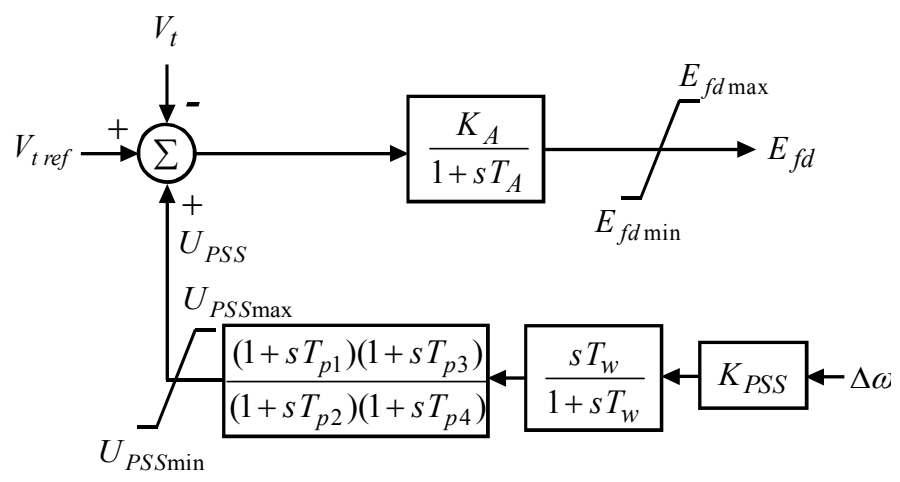

Figure 2. IEEE Type-ST1 excitation system with a PSS

\section{B. Exciter System Modelling}

Here, The IEEE Type-ST1 excitation system is considered which is shown in Figure 2. It represents a bus-fed thyristor excitation system (classified as type STIA1) with an automatic voltage regulator (AVR) and a power system stabilizer (PSS). The dynamic equation is given [2] by:

$$
\dot{E}_{f d}=\frac{1}{T_{A}}\left[K_{A}\left[V_{r e f}-V_{t}+U_{P S S}\right]-E_{f d}\right]
$$

\section{Modelling of FACTS Controllers}

\section{A. TCSC Based Damping Stabilizer}

The design structure of TCSC controller is based on conventional lead-lag structure. The input signal to the controller is speed deviation of the generator. The controller block consists of a gain block, washout block and two stage lead-lag blocks. The controller structure of TCSC is shown in Figure (3). In the controller wash out block act as high pass filter. The phase compensation block is to provide the necessary phase - lead characteristics to compensate for any phase lag between the input and the output signals. The dynamic equation for reactance of TCSC is given by

$$
\stackrel{\bullet}{X_{T C S C}}=\frac{1}{T_{T}}\left[K_{T}\left(X_{T C S C r e f}-U_{T C S C}\right)-X_{T C S C}\right]
$$

\section{B. STATCOM Based Damping Stabilizers}

As shown in Figure 1 the STATCOM is installed at generator terminals of SMIB system which is connected through a transformer with leakage reactance $\mathrm{X}_{\mathrm{S}}$. The STATCOM consists of a 3-phase voltage source converter (VSC) and a DC capacitor whose capacitance is $\mathrm{C}_{\mathrm{DC}}$. The VSC converts DC voltage $\mathrm{V}_{\mathrm{DC}}$ to a controllable $\mathrm{AC}$ voltage $\mathrm{V}_{\mathrm{m}}$ at an angle $\alpha_{\mathrm{s}}$. Voltage difference between these two voltages will provide path for power exchange between STATCOM and the power system. Hence we can maintain power flow by adjusting the voltage magnitude $V_{m}$ and the phase angle $\alpha_{\mathrm{s}}$. From Figure 1 we have [18]

$$
\begin{aligned}
& V_{m}=k m V_{D C}\left(\cos \alpha_{S}+j \sin \alpha_{S}\right)=k m V_{D C} \angle \alpha_{S} \\
& \dot{V_{D C}}=\frac{k m}{C_{D C}}\left(I_{S d} \cos \alpha_{S}+I_{S q} \sin \alpha_{S}\right)
\end{aligned}
$$


where $\mathrm{k}$ is the ratio between AC and DC voltage, $\mathrm{m}$ is modulation index of the PWM and $\alpha_{\mathrm{S}}$ is the firing angle of VSC.

The internal controllers [14] of the STATCOM are designed with PI based controller, which are shown in figures 4 and 5. The Figure 4(a) shows the PI based STATCOM AC voltage controller. The input of the controller is the difference in AC voltage VS and its reference value. Here one more additional input UAS is also taken from the lead- lag based damping controller, which is shown in Figure 4(b). The PI based AC voltage controller gives the modulation index as output, which is having the controller gains $\mathrm{K}_{\mathrm{PAC}}$ and $\mathrm{K}_{\mathrm{IAC}}$. On the other hand, in Figure 5(a) the input given to the PI based DC voltage controller is difference in $\mathrm{DC}$ voltage of the capacitor $\mathrm{V}_{\mathrm{DC}}$ and its reference. DC voltage controller is also having an additional input fed from the damping controller shown in Figure 5(b). The damping controllers of PSS, TCSC and STATCOM are in similar structure such that we can easily coordinate them for better damping.

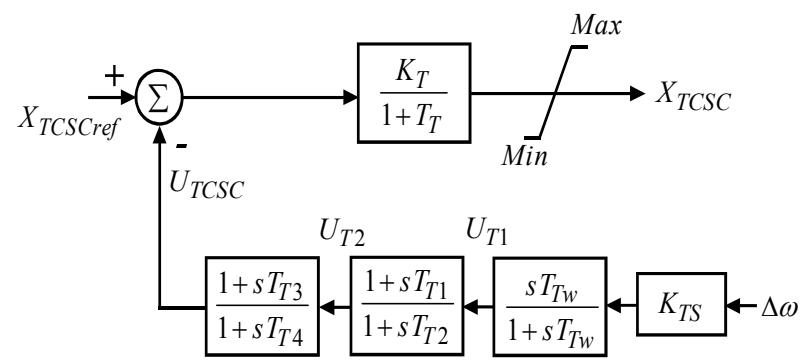

Figure 3. Structure of the TCSC -based controller

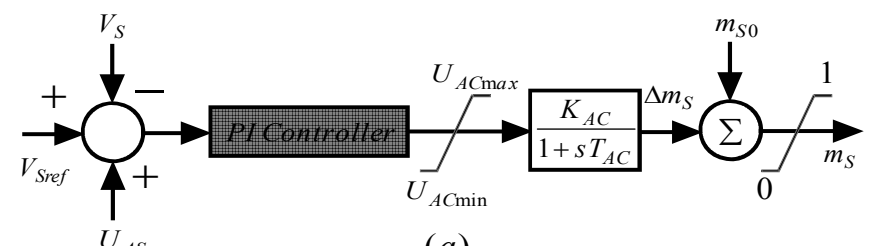

(a)

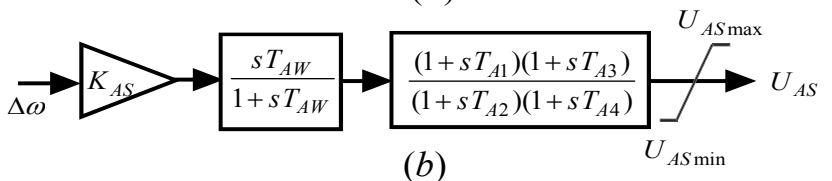

Figure 4. (a) PI based AC voltage controller and (b) additional damping controller of the STATCOM

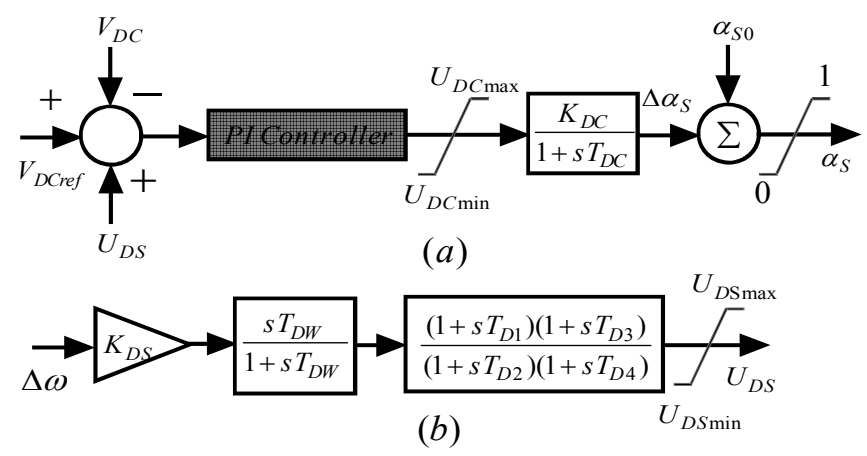

Figure 5. (a) PI based DC voltage controller and (b) additional damping controller of the STATCOM 


\section{Objective Function}

Objective function is a mathematical expression describing a relationship of the optimization parameters that uses the optimization parameters as inputs. In this paper, for optimization of coordinated controller parameters, integral of time-multiplied absolute value of error (ITAE) is considered as objective function. Since integral squired error (ISE) is considered only error and there is no importance is given to time. But for power system stability problems, it is required that settling time should be less and also oscillations should die out soon [19]. However, the main objective is to damp the power oscillations and maintain the voltage profile of the system. This can be achieved by minimizing the value of speed deviation along with terminal voltage variations. So the objective function is formulated with the integration of speed variation and terminal voltage variation. The objective function for SMIB system is given in eq. (8)

$$
J=\int_{0}^{t} t\left[\left|\Delta \omega_{i}\right|+\left|\Delta V_{T i}\right|\right] d t
$$

where $t$ is total simulation time, $\Delta \omega$ is change in speed,and $\Delta V_{T}$ is change in terminal voltage.

In case of multi-machine system the objective is to damp the inter-area speed oscillations. Then, the objective function for multi-machine power system is described in eq. (9)

$$
J=\int_{0}^{t} t\left[\sum\left|\Delta \omega_{I}\right|\right] d t
$$

where $\Delta \omega_{I}$ is the inter-area rotor speed oscillations

So the objective is to minimize ' $J$ ' such that to satisfy the following inequality constraints,

$$
\begin{aligned}
& K_{S}^{\min } \leq K_{S} \leq K_{S}^{\max } \\
& T_{1}^{\min } \leq T_{1} \leq T_{1}^{\max } \\
& T_{2}^{\min } \leq T_{2} \leq T_{2}^{\max } \\
& T_{3}^{\min } \leq T_{3} \leq T_{3}^{\max } \\
& T_{4}^{\min } \leq T_{4} \leq T_{4}^{\max }
\end{aligned}
$$

where $\mathrm{K}_{\mathrm{S}}$ represents gain of damping stabilizer block, and $\mathrm{T}_{1}, \mathrm{~T}_{2}, \mathrm{~T}_{3}, \mathrm{~T}_{4}$ are the time constants of lead-lag blocks in the damping controllers.

\section{Implimentationof Advanced Adaptive Particle Swarm Optimization}

A. Conventional Particle Swarm Optimization: An Over View

Particle swarm optimization is a population based stochastic optimization technique. It was developed by James Kennedy and Russel C Eberhart in 1995[20]. PSO has a flexible and well balanced mechanism to enhance the global and local exploration abilities. Compare to GA, PSO is easy to implement and it consists of only few parameters to adjust. The position and velocity vectors of the $\mathrm{i}^{\text {th }}$ particle in the D-dimensional space can be represented as $X_{i}=\left(x_{i 1}, x_{i 2}, \ldots \ldots \ldots \ldots . . ., x_{i d}\right)$ and $V_{i}=\left(v_{i 1}, v_{i 2}, \ldots \ldots \ldots \ldots . ., v_{i d}\right) \quad$ respectively. The particles in the optimization problem share their information with each other and run towards the best trajectory to find optimum solution in iterative process. In each iteration particles will update their velocities and positions by using the following equations:

$$
\begin{aligned}
& V_{i, \text { iter }+1}=w V_{i, \text { iter }}+c_{1} r_{1}\left(P_{i, \text { iter }}^{\text {best }}-X_{i, \text { iter }}\right)+c_{2} r_{2}\left(G_{i, \text { iter }}^{\text {best }}-X_{i, \text { iter }}\right) \\
& X_{i, \text { iter }+1}=X_{i, \text { iter }}+V_{i, \text { iter }+1}
\end{aligned}
$$


Where $V_{i, \text { iter }}$ and $X_{i, \text { iter }}$ represent the velocity vector and the position vector of $\mathrm{i}^{\text {th }}$ particle at iteration 'iter', $P_{i, \text { iter }}^{\text {best }}$ and $G_{i, \text { iter }}^{\text {best }}$ are personal best position of $i^{\text {th }}$ particle and global best position of swarm in the iteration 'iter'. The constants $c_{1}$ and $c_{2}$ are the positive cognitive and social components that are responsible for varying the particle velocity towards the pbest and gbest, respectively, $r_{1}$ and $r_{2}$ are two random numbers in the range [0-1]. The inertia weight $w$ is responsible for dynamically adjusting the velocity of the particles. To enhance the efficiency of PSO, one can adjust the inertia weight $w$ to linearly reduce during the iterations. The inertia weight is updated by the following equation

$$
w=\left(w_{\max }-w_{\min }\right) \times\left(\frac{\text { iter }_{\max }-\text { iter }}{\text { iter }_{\max }}\right)+w_{\min }
$$

where iter $_{\max }$ is the maximum number of iterations and iter is the current number of iteration. $w_{\max }$ and $w_{\min }$ are maximum and minimum values of inertia weight respectively. The typical range of $w$ from 0.9 at the beginning of the search to 0.4 at the end of the search [21].

\section{B. Advanced Particle Swarm Optimization}

The conventional PSO has a drawback of its inadequate convergence towards global optima. To guarantee the algorithm convergence and avoiding the explosion of the particle swarm (i.e. the state where the particles velocities and positional coordinates careen toward infinity) an advance PSO technique was introduced by Clerc et al. [22]. The advanced PSO hosting a new parameter called constriction factor ' $K$ ' in the velocity equation. Hence, the particles in the swarm can update their velocities and positions by using the following equations:

$$
\begin{aligned}
& V_{i, \text { iter }+1}=K\left[w V_{i, \text { iter }}+c_{1} r_{1}\left(P_{i, \text { iter }}^{\text {best }}-X_{i, \text { iter }}\right)+c_{2} r_{2}\left(G_{i, \text { iter }}^{\text {best }}-X_{i, \text { iter }}\right)\right] \\
& X_{i, \text { iter }+1}=X_{i, \text { iter }}+V_{i, \text { iter }+1}
\end{aligned}
$$

where $K=\frac{2}{\left|2-\varphi-\sqrt{\varphi^{2}-4 \varphi}\right|}, \varphi=c_{1}+c_{2}, \varphi>4$

Usually $c_{1}$ and $c_{2}$ are selected in the range of 0 to 4 .

\section{Advanced Adaptive Particle Swarm Optimization}

In population based optimization methods, the policy is to encourage the individuals to roam through the entire search space without clustering around local optima during the initial stages. However, during latter stages to find the optimum solution efficiently convergence towards the global optima should be encouraged. The concept of time-varying acceleration coefficients (TVAC) $c_{1}$ and $c_{2}$ in addition to time-varying inertia weight factor is introduced in advanced adaptive PSO technique such that AAPSO can efficiently control the local search and provide adequate convergence towards the global optimum solution. During initial stages a large $c_{1}$ and small $c_{2}$ allows the particles to move around search space instead of moving the population best prematurely. At latter stages a small $c_{1}$ and large $c_{2}$ allows the particles to converge towards the global optima. Acceleration coefficients are adaptively changed as follows [23]

$$
\begin{aligned}
& c_{1}=c_{1}^{\text {final }}\left(\frac{\text { iter }}{\text { iter }_{\max }}\right)+c_{1}^{\text {initial }}\left(\frac{\text { iter }_{\max }-\text { iter }}{\text { iter }_{\max }}\right), c_{1}^{\text {final }}<c_{1}^{\text {initial }} \\
& c_{2}=c_{2}^{\text {final }}\left(\frac{\text { iter }}{\text { iter }_{\max }}\right)+c_{2}^{\text {initial }}\left(\frac{\text { iter }_{\max }-\text { iter }}{\text { iter }_{\max }}\right), c_{2}^{\text {final }}>c_{2}^{\text {initial }}
\end{aligned}
$$


Where $c_{1}^{\text {initial }}, c_{2}^{\text {initial }}$ and $c_{1}^{\text {final }}, c_{2}^{\text {final }}$ are initial and final values of the acceleration coefficients $\mathrm{c}_{1}$ and $\mathrm{c}_{2}$ respectively.

In this work, PSO is used for optimizing the control variables of PSS and STATCOM as well as TCSC based controllers. The simultaneous tuning procedure using PSO is given in the form of flow chat shown in Figure 6. Here, all control parameters of damping controllers are optimized by minimizing the objective function given in equation ( 8 or 9). The ranges of optimal parameter are $[0.01-50]$ for Ks, and $[0.05-1]$ for $\mathrm{T}_{1}$ and $\mathrm{T}_{3}$. However, for lead Tcompensation the range of time constants $T_{2}$, T4 should be less than $T_{1}, T_{3}$ so the range taken as [0.001-0.04] for $\mathrm{T} 2$ and $\mathrm{T}_{4}$. The washout time $\mathrm{T}_{\mathrm{w}}$ is set as 5 .

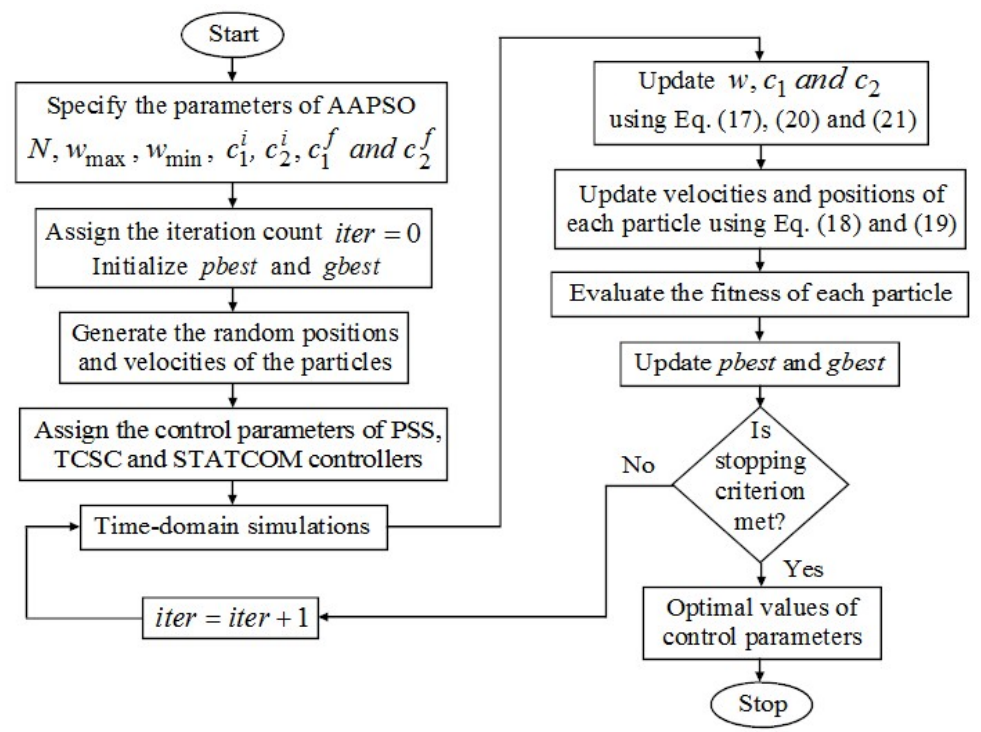

Figure 6. Flow diagram of AAPSO algorithm for proposed coordinated design

\section{Simulation Results}

A. Test system 1: Single machine infinite bus system

The dynamic performance of the test system is analyzed under different loading conditions. The non-linear model simulation is carried out using MATLAB programming for a three phase fault. The fault is applied to the one of the transmission line nearer to the generator terminals. Here, fault applied at $1 \mathrm{sec}$. and cleared at $1.1 \mathrm{sec}$ (i.e. a six cycle fault is applied to the system). The final optimized parameter values of coordinated damping controller are given in Table 1. The following different loadings or changes applied on test system to test the robustness of proposed coordinate controller.

Table 1. Optimal parameter settings of the proposed coordinated controller

\begin{tabular}{|c|c|c|c|c|}
\hline $\begin{array}{c}\text { Control } \\
\text { parameters }\end{array}$ & PSS & TCSC & $\begin{array}{c}\text { STATCOM } \\
\text { ACVC }\end{array}$ & $\begin{array}{c}\text { STATCOM } \\
\text { DCVC }\end{array}$ \\
\hline $\mathrm{K}_{\mathrm{S}}$ & 15.562 & 12.224 & 11.239 & 17.989 \\
\hline $\mathrm{T}_{1}$ & 0.541 & 0.966 & 0.659 & 0.854 \\
\hline $\mathrm{T}_{2}$ & 0.023 & 0.034 & 0.015 & 0.026 \\
\hline $\mathrm{T}_{3}$ & 0.362 & 0.814 & 0.647 & 0.729 \\
\hline $\mathrm{T}_{4}$ & 0.032 & 0.019 & 0.022 & 0.021 \\
\hline
\end{tabular}

Case I: Nominal loading condition (i.e.Pe $=1.0 p . u$. and $Q e=0.25 p . u$.)

The MATLAB simulations results are compared in Figure 7 under nominal loading condition. In the figure (a), (b), (c), and (d) indicate the rotor angle variation, rotor speed variation, active power variation, and terminal voltage variations respectively. From the Figure 
8.(a) we can observe clearly the effectiveness of proposed coordinated controller to damp the rotor angle oscillations. However, Figure 8.(d) indicates how the proposed coordinated controller improves the voltage stability of the test system.

Case II: Heavy loading condition (i.e. $P e=1.1 p . u$. and $Q e=0.3 p$.u.)

The results in Figure 8 show the system dynamic response with different control schemes. From the figure we can observe that the proposed controller under heavy loading condition is effective to damp rotor angle oscillations and enhances the voltage stability of the system.

Case III: Light loading condition (i.e. $P e=0.9 p$.u. and $Q e=0.15 p$.u.)

The system behavior under light loading condition for a six cycle three phase fault is shown in Figure 9. In lightly loading condition also the proposed controller performs better damping to make system stable.
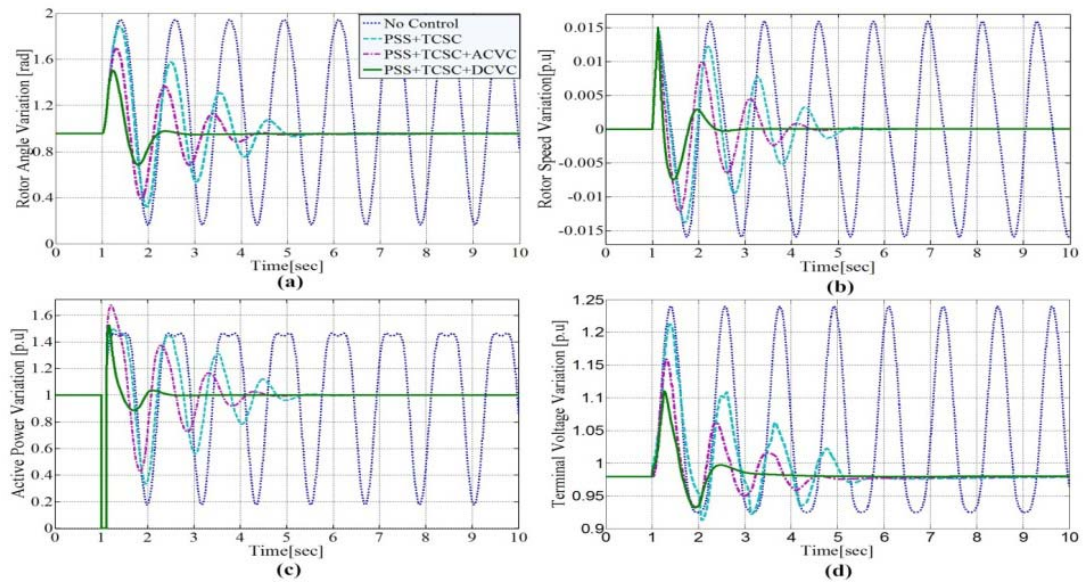

Figure 7. Comparison of simulation results for a six cycle fault disturbance under nominal loading condition. (a) Rotor angle (b) Rotor speed (c) Active power

(d) Terminal voltage variations
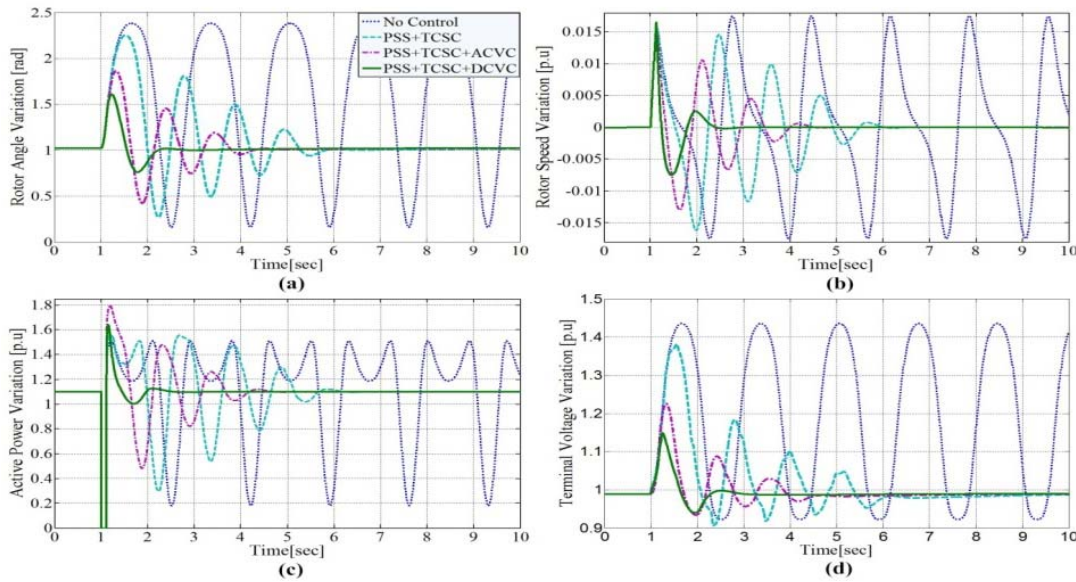

Figure 8. Comparison of simulation results for a six cycle fault disturbance under heavy loading condition. (a) Rotor angle (b) Rotor speed (c) Active power

(d) Terminal voltage variations 

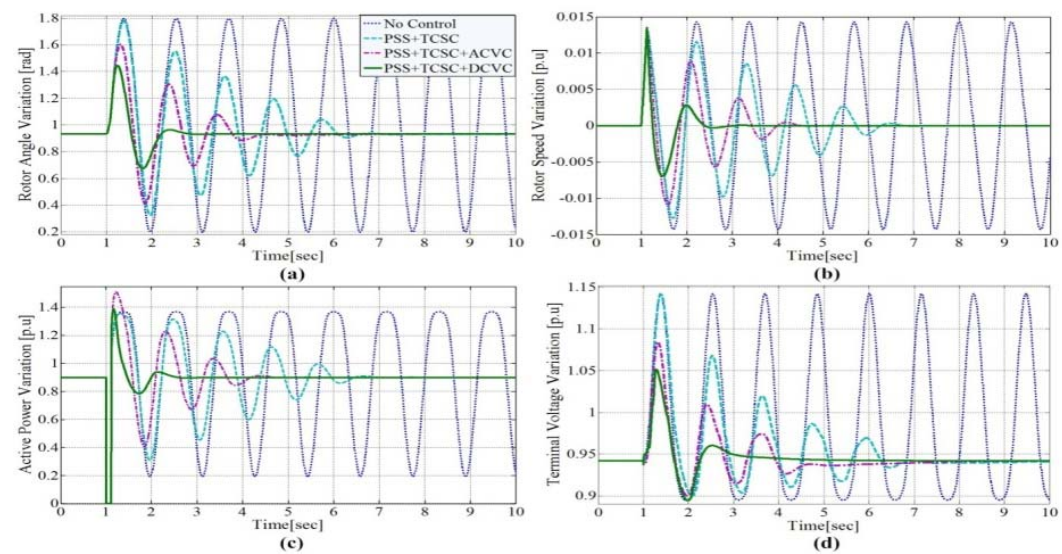

Figure 9. Comparison of simulation results for a six cycle fault disturbance under light loading condition. (a) Rotor angle (b) Rotor speed (c) Active power (d) Terminal voltage variations
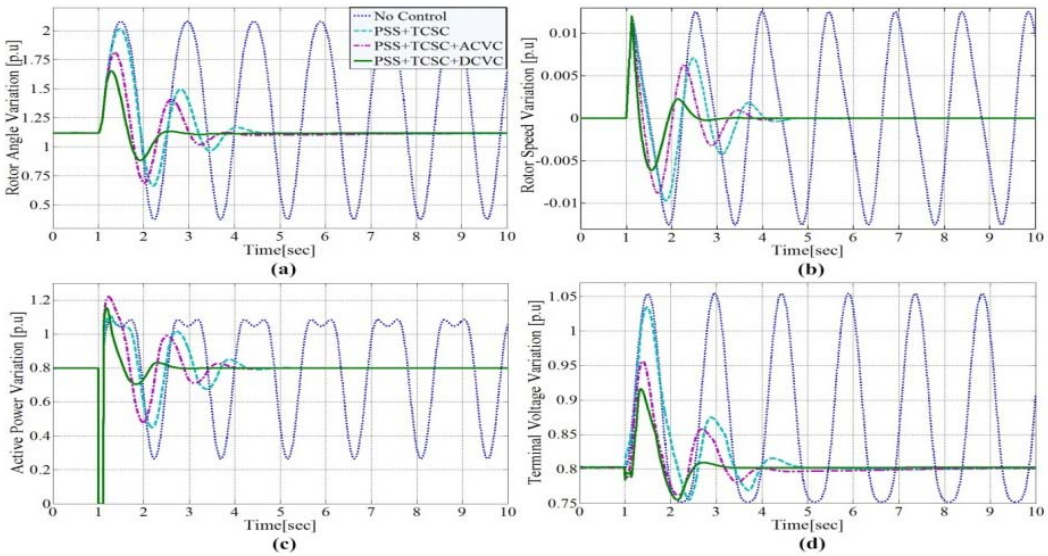

Figure 10. Comparison of simulation results for a six cycle fault disturbance under leadingpower factoropration. (a) Rotor angle (b) Rotor speed (c) Active power

(d) Terminal voltage variations
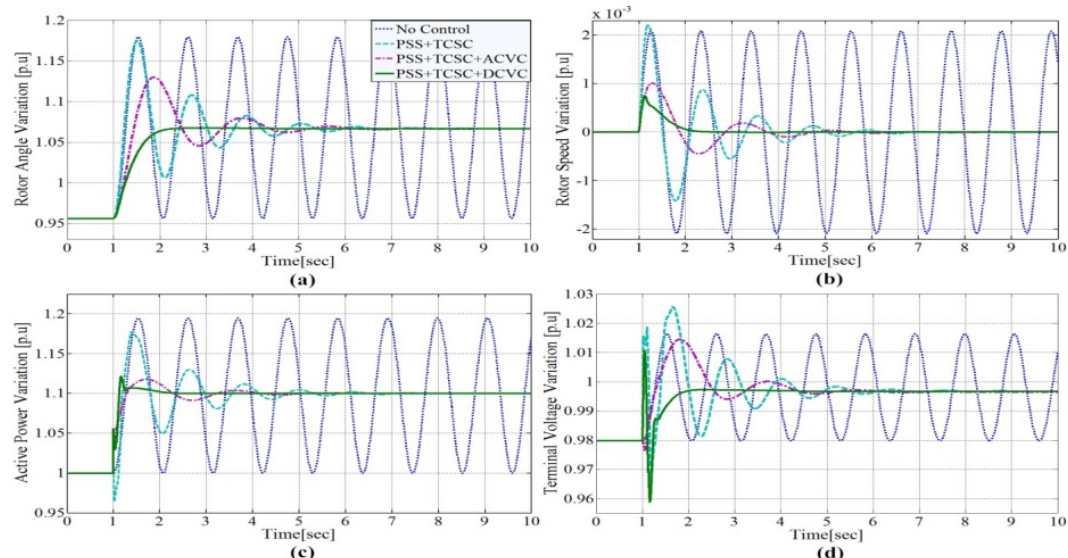

Figure 11. Comparison of simulation results for a six cycle fault disturbance with sudden rise of input power by $10 \%$. (a) Rotor angle (b) Rotor speed (c) Active power

(d) Terminal voltage variations 

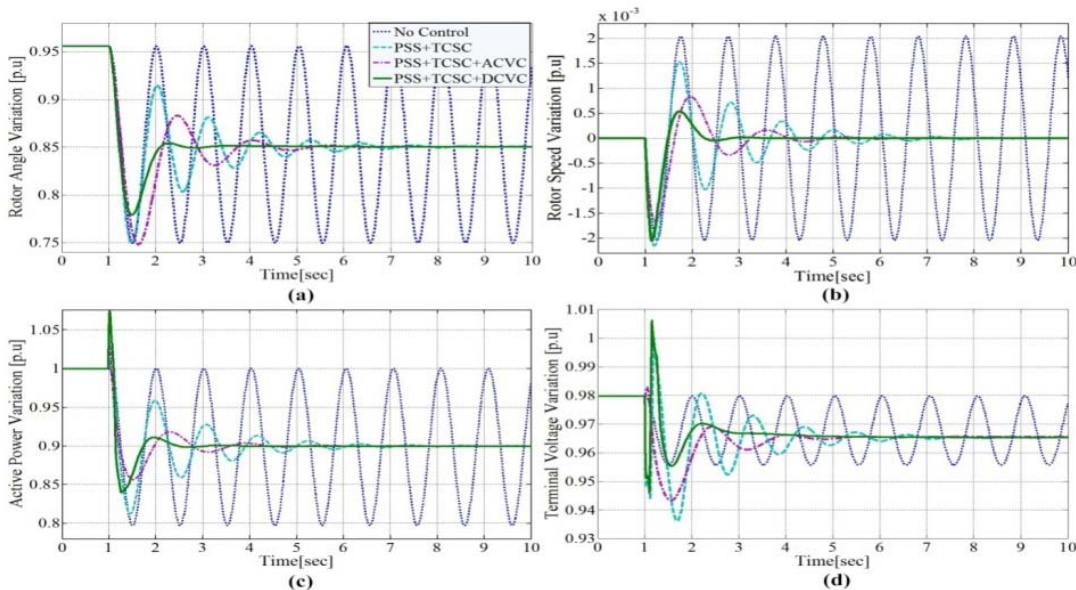

Figure 12. Comparison of simulation results for a six cycle fault disturbance with sudden drop of input power by $10 \%$. (a) Rotor angle (b) Rotor speed (c) Active power

(d) Terminal voltage variations

Case IV: Leading power factor condition (i.e. $P e=0.8 p . u$. and $Q e=-0.1 p . u$.)

Operating the generator under leading power factor causes the drop in generator terminal voltage. However, it increases the rotor angle. The rotor angle as well as terminal voltage of generator with different control schemes is compared in Figure 10. Proposed controller is quite effective in damping rotor angle oscillations and maintains voltage profile.

Case V: $10 \%$ rise in input power (i.e. $P=1.0$ p.u. to 1.1p.u.)

The robustness of proposed controller is tested with a sudden rise of input power by $10 \%$ at time $t=1 \mathrm{sec}$. The dynamic responses of the test system are shown in Figure 11, from the figure it is observe that the test system with proposed controller gives better damping.

Case VI: $10 \%$ drop in input power (i.e. $P=1.0$ p.u. to 0.9 p.u.)

Here, the input power of the generator is dropped by $10 \%$ at time $\mathrm{t}=1 \mathrm{sec}$. The simulation results are compared with different controller schemes in Figure 12. The power oscillations of the test power system are damped out quickly with the proposed coordinated controller.

\section{b. Test system 2: 3-machine 9-bus power system}

In this analysis the 3-machine 9-bus system is considered [3] with PSS installed in all three generators, which is shown in Figure 13.The dynamic performance of the test power system is analyzed over a wide range of operating conditions given in Table 2. Here, the 3-phase fault is applied on line 9-6 with fault starting time at $1 \mathrm{sec}$ and it continues for $100 \mathrm{~ms}$ (i.e six cycle fault). To identify the best location to install TCSC base case power flow analysis is carried out and the results are given in Table 3. From the results, one can clearly observe that the power flow in line 4-9 is high and it is longest line as well. So, we choose the line 4-9 is the best location to install the TCSC in this work. However, the STATCOM is installed at bus 4 which is much affected by the disturbance and it is closer to the large generator $\mathrm{G}_{1}$, which may cause large oscillations even for small disturbances. The AAPSO tuned coordinated control parameters of proposed controller are given in Table 4. Finally, the time-domain simulations are reported for three different loading conditions, namely normal, heavy and light.

\section{Case I: Normal loading condition}

The MATLAB simulation results are compared in Figure 14 under normal loading condition for 3-phase fault disturbance. In the figure (a), (b), and (c) indicates the inter-area speed oscillations $\Delta \omega_{21}, \Delta \omega_{31}$, and $\Delta \omega_{32}$ respectively. The terms ACVC and DCVC indicates 
STATCOM AC voltage controller and STATCOM DC voltage controller respectively in Figure 14-16. Here, four controller schemes are compared in each result. The results show that, the system without controller is unable to damp out the power oscillations. Whereas, the coordinated controller shows better damping effect to power oscillations when compare with other control schemes. The settling time of these oscillations are also very good for the system employed with coordinated controller.

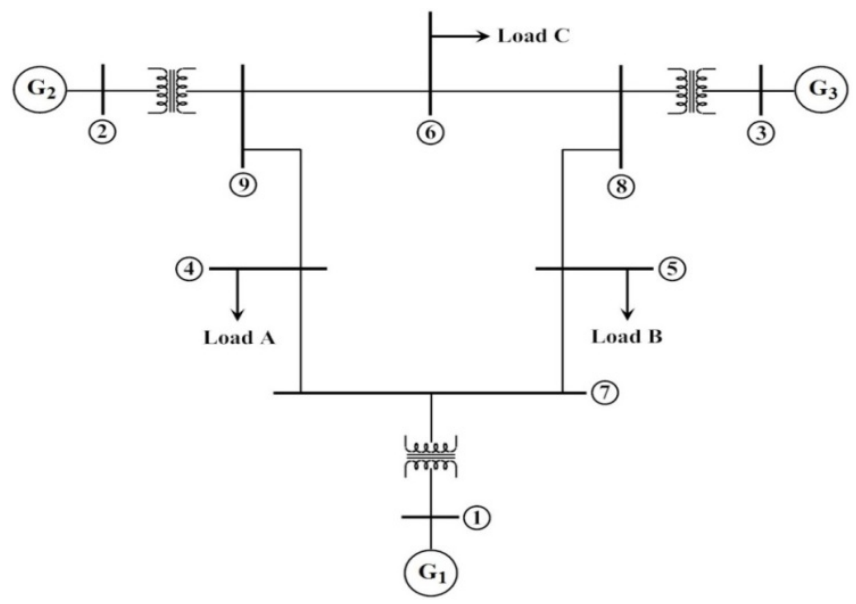

Figure13. 3-machine, 9-bus power system

Table 2. Different loading conditions applied to the test system 2

\begin{tabular}{|c|c|c|c|c|c|c|}
\hline \multirow{2}{*}{} & \multicolumn{2}{|c|}{ Normal } & \multicolumn{2}{c|}{ Heavy } & \multicolumn{2}{c|}{ Light } \\
\cline { 2 - 7 } & $\mathrm{P}$ & $\mathrm{Q}$ & $\mathrm{P}$ & $\mathrm{Q}$ & $\mathrm{P}$ & $\mathrm{Q}$ \\
\hline \multicolumn{7}{|c|}{ Generator } \\
\hline $\mathrm{G}_{1}$ & 0.7326 & 0.2436 & 0.8320 & 0.6178 & 0.5278 & -0.3088 \\
\hline $\mathrm{G}_{2}$ & 1.6300 & 0.0215 & 2.4450 & 0.4248 & 0.8150 & -0.2431 \\
\hline $\mathrm{G}_{3}$ & 0.8500 & -0.1217 & 1.2750 & 0.1373 & 0.4250 & -0.2343 \\
\hline \multicolumn{7}{|c|}{ Load } \\
\hline $\mathrm{A}$ & 1.25 & 0.50 & 1.90 & 0.75 & 0.65 & 0.25 \\
\hline $\mathrm{B}$ & 0.90 & 0.30 & 1.30 & 0.45 & 0.45 & 0.15 \\
\hline $\mathrm{C}$ & 1.00 & 0.35 & 1.50 & 0.50 & 0.50 & 0.17 \\
\hline
\end{tabular}

Table 3. Base case power flow on 100-MVA base.

\begin{tabular}{|c|c|c|}
\hline From bus & To Bus & Real power flow (p.u) \\
\hline 7 & 4 & 0.2766 \\
\hline 4 & 9 & 0.9812 \\
\hline 9 & 6 & 0.6178 \\
\hline 6 & 8 & 0.3893 \\
\hline 8 & 5 & 0.4589 \\
\hline 5 & 7 & 0.4526 \\
\hline
\end{tabular}

Table 4. Optimal parameters of different controllers for coordianted design

\begin{tabular}{|c|c|c|c|c|c|c|}
\hline $\begin{array}{c}\text { Control } \\
\text { parameters }\end{array}$ & PSS1 & PSS2 & PSS3 & TCSC & $\begin{array}{c}\text { STATCOM } \\
\text { ACVC }\end{array}$ & $\begin{array}{c}\text { STATCOM } \\
\text { DCVC }\end{array}$ \\
\hline $\mathrm{K}_{\mathrm{S}}$ & 24.2340 & 7.5122 & 8.2341 & 1.2814 & 3.1274 & 5.5037 \\
\hline $\mathrm{T}_{1}$ & 0.6521 & 0.4312 & 0.5012 & 0.3612 & 0.2348 & 0.4223 \\
\hline $\mathrm{T}_{2}$ & 0.0321 & 0.0289 & 0.0388 & 0.0217 & 0.0372 & 0.0202 \\
\hline $\mathrm{T}_{3}$ & 0.7124 & 0.5088 & 0.6127 & 0.2890 & 0.4089 & 0.2711 \\
\hline $\mathrm{T}_{4}$ & 0.0327 & 0.0250 & 0.0361 & 0.0229 & 0.0211 & 0.0370 \\
\hline
\end{tabular}




\section{Case II: Heavy loading condition}

To test the robustness of proposed coordinated controller the test system is operated under heavy loading condition. In heavy load condition the simulations are carried out with the active power values of generators $G_{2}, G_{3}$ and all loads A, B, C are increased $50 \%$ more than that of normal load condition. The Figure 15 shows the performance of test system for heavy loading. From the results, the test system without controller is unstable and the generators are losing their synchronism. However, the test system with coordinated controller shows better damping as well as adequate settling time compared with other control schemes. In heavy loading condition also the proposed controller performs well in all accepts.

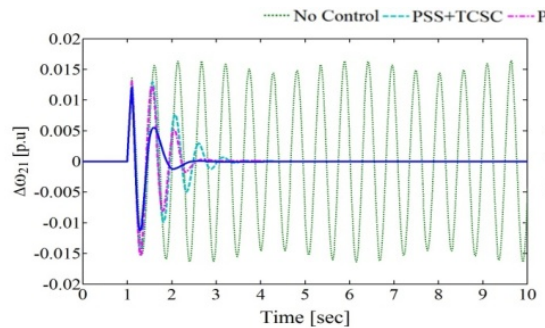

(a)

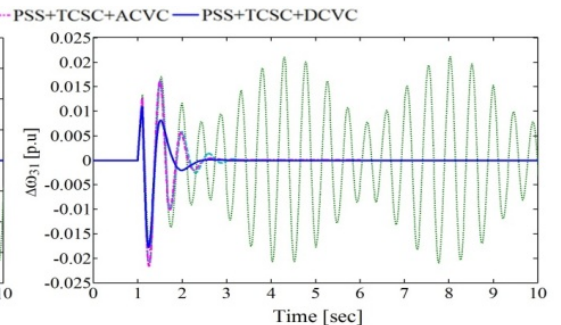

(b)

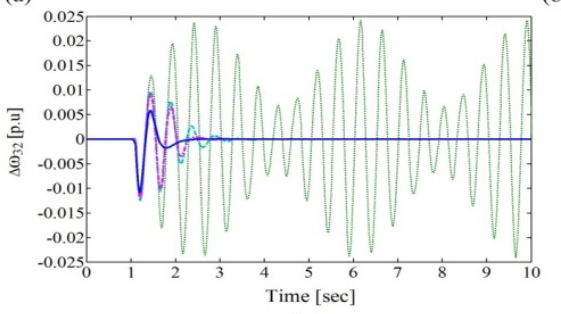

(c)

Figure 14. System performance for a six cycle fault under normal loading.

(a) $\Delta \omega_{21}$ (b) $\Delta \omega_{31}$ (c) $\Delta \omega_{23}$.

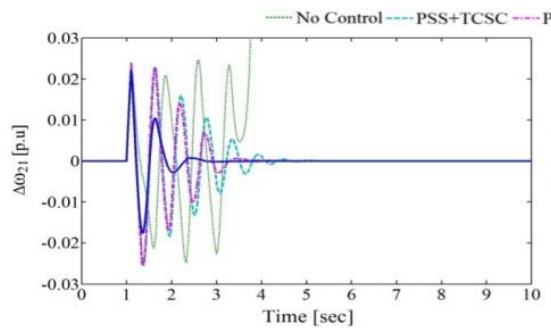

(a)

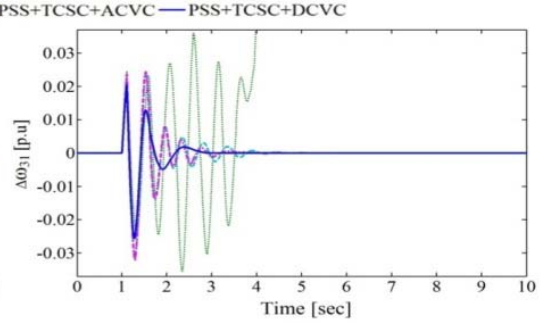

(b)

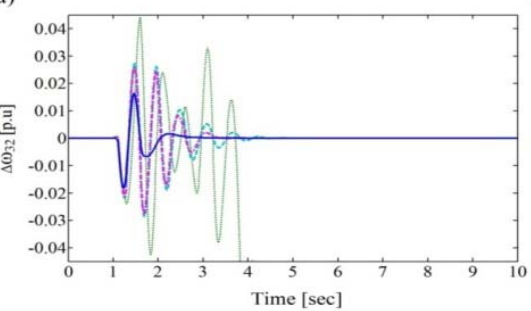

(c)

Figure 15. System performance for a six cycle fault under heavy loading.

(a) $\Delta \omega_{21}$ (b) $\Delta \omega_{31}$ (c) $\Delta \omega_{23}$. 


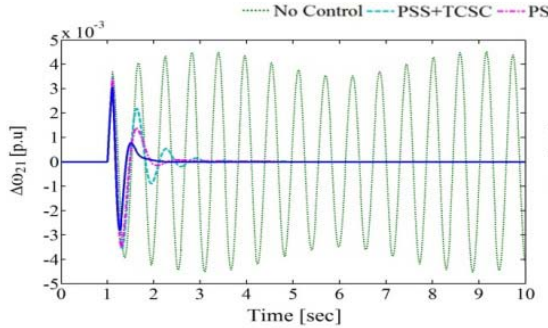

(a)

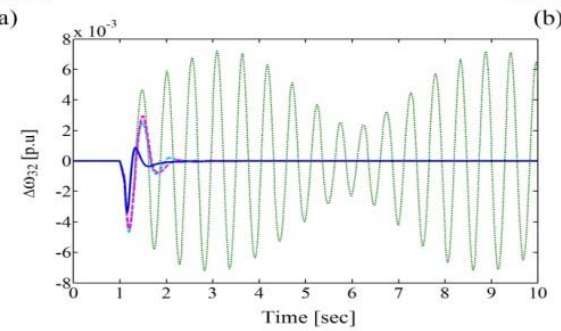

(c)

Figure 16. System performance for a six cycle fault under light loading.

(a) $\Delta \omega_{21}$ (b) $\Delta \omega_{31}$ (c) $\Delta \omega_{23}$.

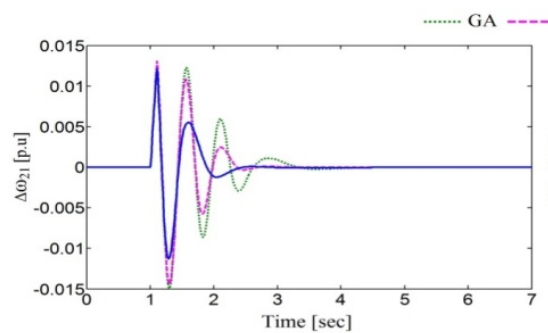

(a)

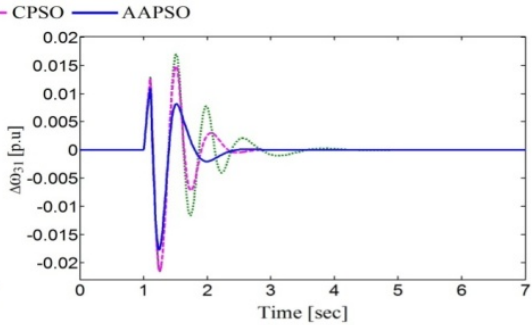

(b)

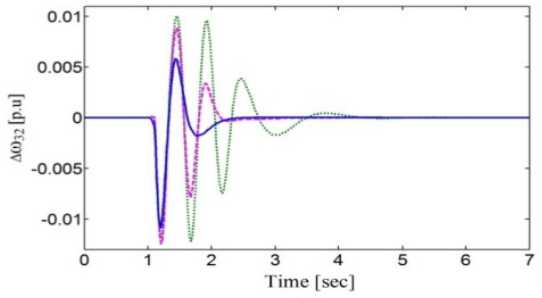

(c)

Figure 17. System performance for a six cycle fault with different optimization algorithms

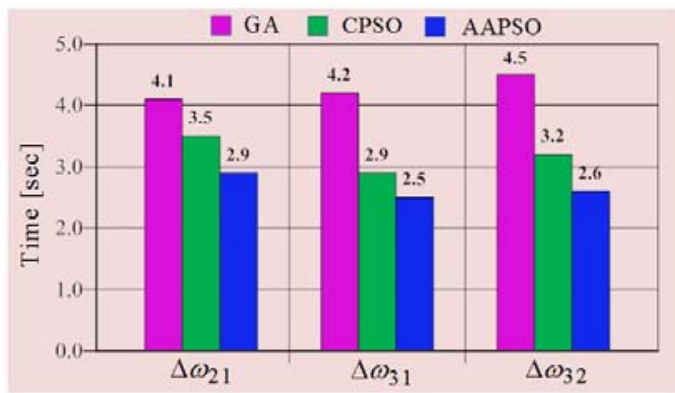

Figure 18. Comparison of settling times of system responses with different optimization algorithms 


\section{Case III: Light loading condition}

The Figure 16 shows the performance of test system for light loading. In light load condition the simulations are carried out with the active power values of generators $G_{2}, G_{3}$ and all loads A, B, C are decreased $50 \%$ less than that of normal load condition. It is found that in light loading condition, all generators are operated in leading power factor. In this operating condition the power oscillations damp out quickly with the proposed coordinated controller compared to the other controller schemes. On the other hand, the test system with no controller is unable to damp out these oscillations. Hence, the robustness of proposed coordinated damping controller is tested in three different loading conditions. It provides acceptable damping effect and adequate settling time for power oscillations under severe disturbance.

Case IV: Proposed Controller design using different optimization algorithms

The proposed coordinated controller design is obtained using different optimization techniques such as GA, Conventional PSO (CPSO) and AAPSO. To operate all these optimization algorithms at equal complexities, we considered 100 iterations/generations and each of it consists of 100 individuals. The comparison of different optimization algorithms in designing the coordinated controller is shown in Figure 17 for a six cycle fault under normal loading condition. The comparison of settling time of the oscillations is shown in Figure 18. From the results, it is found that the AAPSO based coordinated controller design is more effective in damping oscillations and it provides very low settling time comparing with other conventional optimization algorithms.

\section{Conclusions}

In this paper, optimized coordinated control of a power system stabilizer (PSS) with TCSC and STATCOM based damping controllers are discussed. Here two internal controller of STATCOM is taken namely AC voltage controller and DC voltage controller. An objective function is minimized using AAPSO for finding the optimal control parameters of coordinated controller. Four different control schemes are employed on the test system to investigate the performance of the proposed controller. The time domain simulation of a non-linear system is carried out in MATLAB software package. Here, SMIB as well as multi-machine power system are examined for analysis. The robustness of the proposed coordinated controller is investigated by testing its performance under different loading conditions. The simulation results show that the test systems dynamic performance and overall damping effect are enhanced by simultaneous tuning of all three damping controllers. The AAPSO based coordinated controller design outperforms the other optimization techniques like GA and CPSO. Therefore, coordinated control of PSS and FACTS based stabilizers provides better damping of power oscillations and maintains system stability as well.

\section{References}

[1] Prabha Kundur, John Paserba, Venkat Ajjarapu, GOran Andersson, Anjan Bose, Claudio Canizares, Nikos Hatziargyriou, David Hill, Alex Stankovic, Carson Taylor, Thierry Van Cutsem, Vijay Vittal, Definition and Classification of Power System stability, IEEE Trans. Power Syst., 19 (2004) 1387-1401.

[2] P. Kundur, Power System Stability and Control, McGraw-Hill, New York, 1994.

[3] P. M. Anderson, A. A. Fouad, Power system control and stability. Wiley-Interscience, Piscataway, N.J, 2003.

[4] G. Hingorani, L. Gyugyi, "Understanding FACTS-Concepts and Technology of Flexible AC Transmission Systems", IEEE Press, New York,2000.

[5] N. Mithulananthan, C. A. Canizares, J. Reeve, G. J. Rogers, "Comparison of PSS, SVC, and STATCOM Controllers for Damping Power System Oscillations", IEEE Trans. Power Syst., 18 (2003) 786 - 792.

[6] J. Kennedy, and R. Eberhart, "Particle Swarm Optimization," IEEE International Conference on Neural Networks, vol. 4, pp. 1942-1948, November 1995. 
[7] M. A. Abido, "Optimal Design of Power-System Stabilizers Using Particle Swarm Optimization" IEEE Trans. Energy Conv., vol. 17, no. 3, pp. 406-413, September 2002.

[8] H. Shayeghi, H.A. Shayanfar, A. Safari, and R. Aghmasheh, "A robust PSSs design using PSO in a multi-machine environment" Energy Convers Manage, vol. 51, pp. 696-702, 2010.

[9] M. A. Abido, "Analysis of Power System Stability Enhancement via Excitation and Facts-Based Stabilizers" Elect. Power Compon and Syst., vol. 32, no. 1, pp. 75-91, 2004.

[10] Sidhartha Panda, and Narayana Prasad Padhy, "Comparison of particle swarm optimization and genetic algorithm for FACTS-based controller design" Applied Soft Computing, vol. 8, pp. 1418-1427, 2008.

[11] Xianzhang Lei, Edwin N. Lerch, Dusan Povh, "Optimization and Coordination of Damping Controls for Improving System Dynamic Performance", IEEE Trans. Power Syst., 16 (2001) 473-480.

[12] Y.L. Abdel-Magid, M.A. Abido, Robust coordinated design of excitation and TCSCbased stabilizers using genetic algorithms, Electric Power Syst. Res., 69 (2004) 129-141.

[13] E.S. Ali, S.M. Abd-Elazim, "Coordinated design of PSSs and TCSC via bacterial swarm optimization algorithm in a multimachine power system", Int. J. Elect. Power and Energy Syst., 36 (2012) 84-92.

[14] H.F.Wang, Interactions, 'Multivariable Design of STATCOM AC and DC Voltage Control", Int. J. Elect. Power and Energy Syst., 25 (2003) 387-394.

[15] W. L. Chen, Y. G. Huang, C. H. Pien, "Control and Performance Analysis for a Capacitor-coordinated Static Synchronous Compensator to Enhance Dynamic Compensation Capability", Elect. Power Compon. and Syst., 39 (2011) 991-1006.

[16] T.T. Nguyen, R. Gianto, "Optimal design for control coordination of power system stabilisers and flexible alternating current transmission system devices with controller saturation limits", IET Gener. Transm. Distrib., 4 (2010) 1028 - 1043.

[17] M. Aliakbar Golkar, M. Zarringhalami, "Coordinated Design of PSS and STATCOM Parameters for Power System Stability Improvement Using Genetic Algorithm", Iranian J. of Elect.and Computer Engg., 8 (2009) 80-88.

[18] H. F. Wang, "Phillips-Heffron model of power systems installed with STATCOM and applications", IEE Proc-Gener. Transm. Distrib., 146 (1999) 521-527.

[19] Sidhartha Panda, "Differentital evolutionary algorithm for TCSC-based controller design", Simulation Modelling Practice and Theory, 17 (2009) 1618-1634.

[20] K.T. Chaturvedi, M. Pandit, L. Srivastava. "Self-organizing hierarchical particle swarm optimization for non-convex economic dispatch". IEEE Trans. Power Syst. 2008; 23(3):1079-1087.

[21] Yu-hui Shi, Russell C Eberhart. "Empirical study of particle swarm optimization". in Proc. IEEE Int. Congr. Evolutionary Computation 1999; 3:101-106.

[22] M. Clerc, J. Kennedy. "The particle swarm - explosion, stability, and convergence in a multidimensional complex space". IEEE Trans. Evolutionary Computation, 2002; 6:58-73.

[23] A. Ratnaweera, S.K. Halgamuge, H.C. Watson. "Self-organizing hierarchical particle swarm optimizer with time-varying acceleration coefficients". IEEE Trans. Evol. Comput. 2004; 8(3):240-255.

\section{Appendix A}

a). The SMIB system parameters:

All the data are in per unit unless specified.

Genarator $\quad: \mathrm{H}=8.0 \mathrm{MJ} / \mathrm{MVA}, \mathrm{D} 1=0.0, \mathrm{~T}_{\mathrm{d} 0}^{\prime}=5.044 \mathrm{sec} ., \mathrm{f}=60 \mathrm{~Hz}$

Transformer $\quad: \mathrm{X}_{\mathrm{T}}=0.1, \mathrm{X}_{\mathrm{S}}=0.1$

$$
\mathrm{X}_{\mathrm{d}}=0.9, \mathrm{X}_{\mathrm{q}}=0.6, \mathrm{X}_{\mathrm{d}}^{\prime}=0.3
$$

Transmission lines : $\mathrm{X}_{\mathrm{L} 1}=\mathrm{X}_{\mathrm{L} 2}=0.3$ 
b). System data for 3-machine 9-bus power system [3]: all data in p.u. on a 100-MVA base

$$
\begin{aligned}
& H_{1}=23.64, H_{2}=6.40, H_{3}=3.01, X_{d 1}=0.1460, X_{d 2}=0.8958, X_{d 3}=1.3125, X_{d 1}^{\prime}=0.0608, \\
& X_{d 2}^{\prime}=0.1198, X_{d 3}^{\prime}=0.1813, X_{q 1}=0.0969, X_{q 2}=0.8645, X_{q 3}=1.2578, T_{d 01}^{\prime}=8.96, \\
& T_{d 02}^{\prime}=6.00, T_{d 03}^{\prime}=5.89 \\
& \text { Exciter: } K_{A 1}=K_{A 2}=K_{A 3}=20, T_{A 1}=T_{A 2}=T_{A 3}=0.05 \mathrm{~s} .
\end{aligned}
$$

c). Optimization parameters:

Genetic Algorithms : Selection operator: Roulette Wheel, Crossover Probability $=0.8$, Mutation Probability $=0.01$, Termination Method: Maximum Generations.

Conventional PSO $\quad \mathrm{C}_{1}=\mathrm{C}_{2}=2.0, w=0.9$, Termination Method: Maximum iterations.

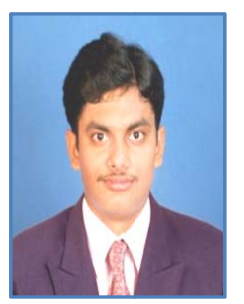

Rajendraprasad Narne was born in Andhra Pradesh, India on November 21 1985. He received his B.Tech. degree in Electrical and Electronics Engineering from JNTU Hyderabad, India, in 2007, and the M .E. Degree in Power System Engineering from Anna University, India in 2009. Currently, he is working towards his Ph.D. degree at National Institute of Technology, Rourkela, Orissa, India, under the supervision of Prof. P.C.Panda. His research interests include power system stability and control, and FACTS controllers.

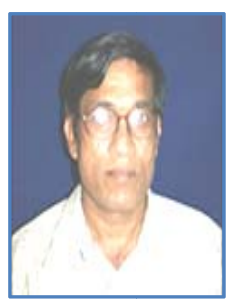

Prafulla Chandra Panda (SM'05) was born in Orissa on November 01, 1948. He received his B.Sc., M.Sc., and Ph.D. degrees, all in electrical engineering from Sambalpur University, Orissa, India, in the year 1971, 1974 and 1990 respectively.

Since 1977, he has been with National Institute of Technology, Rourkela, Orissa, India, where he is now a professor. He is senior member of IEEE and Fellow of IE (India). At present, his research interests include Power system dynamic stability analysis, FACTS Controllers and High voltage dc

transmission. 